\title{
Consistency of Care and Blood Pressure Control among Elderly African Americans and Whites with Hypertension
}

\author{
Daniel L. Howard, PhD, April P. Carson, PhD, DaJuanicia N. Holmes, MS, \\ and Jay S. Kaufman, $P h D$
}

Objective: To determine whether racial differences exist between consistency of medical care and blood pressure (BP) control over time among elderly, hypertensive African Americans and whites.

Design: Participants included 1402 African Americans and 1058 whites from the Piedmont Health Survey of the Elderly who were hypertensive (systolic BP $\geq 140 \mathrm{~mm} \mathrm{Hg}$, diastolic BP $\geq 90 \mathrm{~mm} \mathrm{Hg}$, or used antihypertensive medications) at baseline (in 1987). Consistency of care was assessed based on self-reported receipt of physician care at each wave and categorized as consistent (care at each wave); inconsistent (care at some, but not all waves); or no standard care (no care at any wave). BP control was defined as systolic BP $<140 \mathrm{~mm} \mathrm{Hg}$ and diastolic BP $<90 \mathrm{~mm} \mathrm{Hg}$ at subsequent waves of participation (in 1990, 1994, and 1998). Repeated measures regression was used to longitudinally assess the association between consistency of care and BP control.

Results: African Americans had a less favorable health profile and significantly less consistency of care over time $(P<.0001)$. In analyses adjusted for demographic factors, participants with consistent or inconsistent care had greater odds of BP control (odds ratio, 1.34; 95\% CI, 1.09-1.64 and odds ratio, $1.41 ; 95 \%$ CI, 1.12-1.78, respectively) than those with no standard care, but these associations were attenuated after additional adjustment for health care characteristics and comorbidities.

Conclusions: Compared with no standard care, receipt of consistent or inconsistent physician care was associated with BP control among the elderly. These associations did not differ by race, although African Americans were more likely to report inconsistent or no standard care, which suggests that disparities in health care access remain. (J Am Board Fam Med 2009;22:307-15.)

Racial disparities in hypertension prevalence and its sequelae persist in the United States. ${ }^{1-3}$ African Americans are disproportionately affected by hypertension and, when afflicted, have less than optimal BP control despite more than 3 decades of evidence about the benefits of pharmacologic ther-

This article was externally peer reviewed.

Submitted 11 July 2008; revised 6 November 2008; accepted 24 November 2008.

From the Institute for Health, Social, and Community Research, Shaw University, Raleigh, NC (DLH, APC, DNH); and the Department of Epidemiology, School of Public Health, University of North Carolina at Chapel Hill (JSK).

Funding: The study was funded by the Department of Health and Human Services Agency for Healthcare Research and Quality grant \#R24 HS013353. Investigators were also supported, in part, by the National Institutes of Health National Center on Minority Health and Health Disparities grant \#P60 MD000239 and National Center for Research Resources grant \#C06 RR020139.

Conflict of interest: none declared.

Corresponding author: Daniel L. Howard, PhD, Professor and Director, The Institute for Health, Social, and Community Research at Shaw University, 118 E. South Street, Raleigh, NC 27601 (E-mail: howardd@shawu.edu). apy. ${ }^{4}$ Several factors have been postulated to contribute to racial disparities in hypertension prevalence and control, including diet, physical activity, health insurance, and access to quality health care. ${ }^{5}$

Consistency of care, a measure of access to care and the health care relationship over time between patients and physicians, is an important factor in the prevention and treatment of chronic diseases, including hypertension. ${ }^{6}$ A previous study reported that consistency of care was associated with hypertension diagnosis and receipt of antihypertensive therapy among the elderly, but did not evaluate blood pressure (BP) control. ${ }^{7}$ In another prospective study of elderly adults, BP control was comparable between African Americans and whites during a 9-year study period, but consistency of care was not evaluated. ${ }^{8} \mathrm{~A}$ cross-sectional study reported an association between usual provider and BP control among the general population, ${ }^{5}$ but to date no study has examined patterns of consistency of care and BP control over time specifically among elderly hypertensive patients. Investigation of these pat- 
terns may provide useful information about potential racial differences in long-term health care trends and outcomes among those who have hypertension. Thus, the purpose of this populationbased, observational study was to describe and examine the relationship between consistency of care and BP control among elderly African Americans and whites during a 12 -year period.

\section{Methods}

\section{Study Design}

The Piedmont Health Survey of the Elderly (PHSE) was conducted by the Duke University Center for Aging and Human Development to assess health, well-being, morbidity, and mortality in community-dwelling older populations as part of the Established Populations for the Epidemiologic Study of the Elderly. A detailed description of the Established Populations for the Epidemiologic Study of the Elderly study design has been published. ${ }^{9}$ The PHSE selected 5226 participants aged 65 years and older at baseline (1987) from 5 counties in North Carolina (1 urban, 4 rural), with an over-sampling of African Americans. Trained personnel collected baseline measures for 4162 elders ( $80 \%$ response rate) through 90 -minute in-home interviews; participants had 3 in-person follow-up examinations (in 1990, 1994, and 1998) and also received annual telephone contacts in the intermittent years.

\section{Study Sample}

This study excluded participants who were not white or African-American ( $\mathrm{n}=26)$; were younger than 65 years old $(\mathrm{n}=3)$; did not participate in at least one follow-up wave $(\mathrm{n}=540)$; were not hypertensive ( $\mathrm{n}=1044)$; or were missing BP measurements $(\mathrm{n}=89)$. After these exclusions, 1402 African American and 1058 white participants who were hypertensive at baseline (systolic BP $\geq 140$ $\mathrm{mm} \mathrm{Hg}$, diastolic $\mathrm{BP} \geq 90 \mathrm{~mm} \mathrm{Hg}$, or currently taking antihypertensive medications) and had at least one additional wave of data were included in this study.

\section{Outcome Variables}

Following a standardized protocol, BP measurements were taken at baseline and each subsequent wave. Two sitting BP measurements were taken and the mean value was used to determine $\mathrm{BP}$ control status: controlled (systolic BP $<140 \mathrm{~mm} \mathrm{Hg}$ or diastolic BP $<90 \mathrm{~mm} \mathrm{Hg}$ ) and uncontrolled (systolic BP >140 $\mathrm{mm} \mathrm{Hg}$ or diastolic $\mathrm{BP}>90 \mathrm{~mm} \mathrm{Hg}$ ).

\section{Predictor Variable}

Consistency of care was defined as the level of consistent medical care received from a physician, which was derived from a PHSE survey question about the use of health care services. During each of the 4 waves, respondents were asked the following question: "When you want help with or care for a [physical] health problem, where do you usually go?" Responses included nowhere, no usual place, a physician, a hospital, or other. For those who responded a physician, they were asked the physician's name and where did they see the doctor-in a private office, or clinic, a public clinic, a Veterans' Affairs hospital, other hospital, or where. Consistency of care was classified into 3 categories: consistent care (receipt of physician care across all waves of participation); inconsistent care (receipt of physician care across some, but not all, waves of participation); and no standard care (no receipt of physician care across any waves of participation). The participant was not required to have the same physician at each wave of participation, but was required to have a named physician at each wave of participation to be counted as having received care at that wave. If a participant was counted as having received care by a named physician, then the location of the care was then assessed as occurring at a private practice, clinic, hospital, or other institution.

\section{Covariates}

The sociodemographic variables assessed were age at baseline, sex, education, annual income, marital status, and residence in a rural area. General health perception (excellent, good, fair, or poor); history of chronic diseases (heart disease, cancer, stroke, and diabetes); and limitations in activities of daily living (ADLs) were also ascertained. Several health care variables were assessed, including Medicaid (a government health insurance program for low-income persons); Medigap (private supplemental health insurance for Medicare beneficiaries for health care costs that are not covered); residence in a nursing home; residence in the county where care was received; location of care (clinic/hospital, private practice, or other); and satisfaction with care. Lifestyle variables such as diet, physical activity, 
alcohol consumption, and smoking were also collected; however, these variables were not collected over all 4 waves and/or a large proportion of responses were missing, so these variables were not included in the analyses.

Participants were asked to bring in the containers for all the medications that they had taken during the previous 2 weeks or that they take as necessary. Names of the medications were recorded and classified by indication. For antihypertensive therapy, medication included single-agent therapy (diuretics, beta-blockers, calcium channel blockers, angiotensin-converting enzyme inhibitors, or other antihypertensive agents) and combination therapy (diuretics plus other antihypertensive agents).

\section{Analysis}

Analyses of variance, $\chi^{2}$, and $t$ tests were used to describe and compare, across 4 waves, consistency of care and BP control between African Americans and whites. Repeated measures models were fit using generalized estimating equations to simultaneously test for racial differences and trends across time for each of the sociodemographic, health status, health care, and consistency of care variables. ${ }^{10}$

To test whether there was a difference in the association between consistency of care and BP control across time, multivariable repeated measures models were fit using generalized estimating equations. The exposure was categorized into 3 levels: no standard care (referent group), inconsistent care, and consistent care. Crude and adjusted analyses assessed the association between consistency of care and BP control. Age, sex, and education were measured at baseline and did not change over time whereas annual income, work status, marital status, history of chronic diseases, limitation in ADLs, health insurance coverage, residence in a nursing home, satisfaction with care, and antihypertensive medication use were included in the models as time-varying covariates. A sensitivity analysis was also done to assess the effect of loss to follow-up at each wave. Loss to follow-up increased during the 12 -year study period: $2 \%$ from wave 1 to wave $2 ; 20 \%$ from wave 2 to wave 3 ; and $43 \%$ from wave 3 to wave 4 . Because of the increase in the loss to follow-up from wave 3 to wave 4 , the sociodemographic characteristics of those who participated in both wave 3 and wave 4 were compared with those who participated in wave 3 , but not wave
4, with differences noted for education, income, marital status, and working status. A sensitivity analysis was done to assess potential differences in effect estimates using data from all waves (1-4) and data from waves 1 to 3 only. The estimates were comparable, and therefore all waves of data (waves 1-4) were used in this analysis. All analyses were done using version 9.1 of SAS software (SAS Institue, Inc., Cary, NC).

\section{Results}

During the baseline examination, 31\% of the participants were men, $57 \%$ were African-American, and the average age was 73 years (Table 1). The majority of participants had an annual income between $\$ 4,000$ and $\$ 15,000$ and were not working. Compared with whites over time, African Americans had fewer mean years of education $(P<.001)$, were less likely to be currently married $(P<.001)$, and more likely to reside in a rural area $(P<.001)$. Most participants reported excellent or good health, although there was a significant trend in health status over time, with fewer African Americans reporting excellent or good health $(P<.001)$. For diagnosed health conditions during the 4 waves, a greater proportion of whites than African Americans reported having cancer $(P<.001)$ or a heart condition $(P=.0493)$. In contrast, a greater proportion of African Americans reported having diabetes $(P<.001)$ whereas no racial difference over time was noted for the diagnosis of stroke. Limitations to ADLs increased during the 4 waves, with African Americans reporting more limitations than whites $(P=.0139)$. The proportion of participants with Medicaid insurance increased over time, although no clear pattern was observed for Medigap supplemental insurance coverage. More African Americans had Medicaid coverage whereas more whites had Medigap supplemental insurance coverage (both $P<.001$ ); more African Americans received care from a public clinic whereas more white participants received care from a private practice.

The distribution of consistency of care across the 4 waves is presented in Table 2. The majority of the study sample had consistent care; significantly more whites had consistent care $(P<.001)$ and more African Americans had inconsistent $(P<$ $.001)$ or no standard care $(P<.001)$. Across all 4 


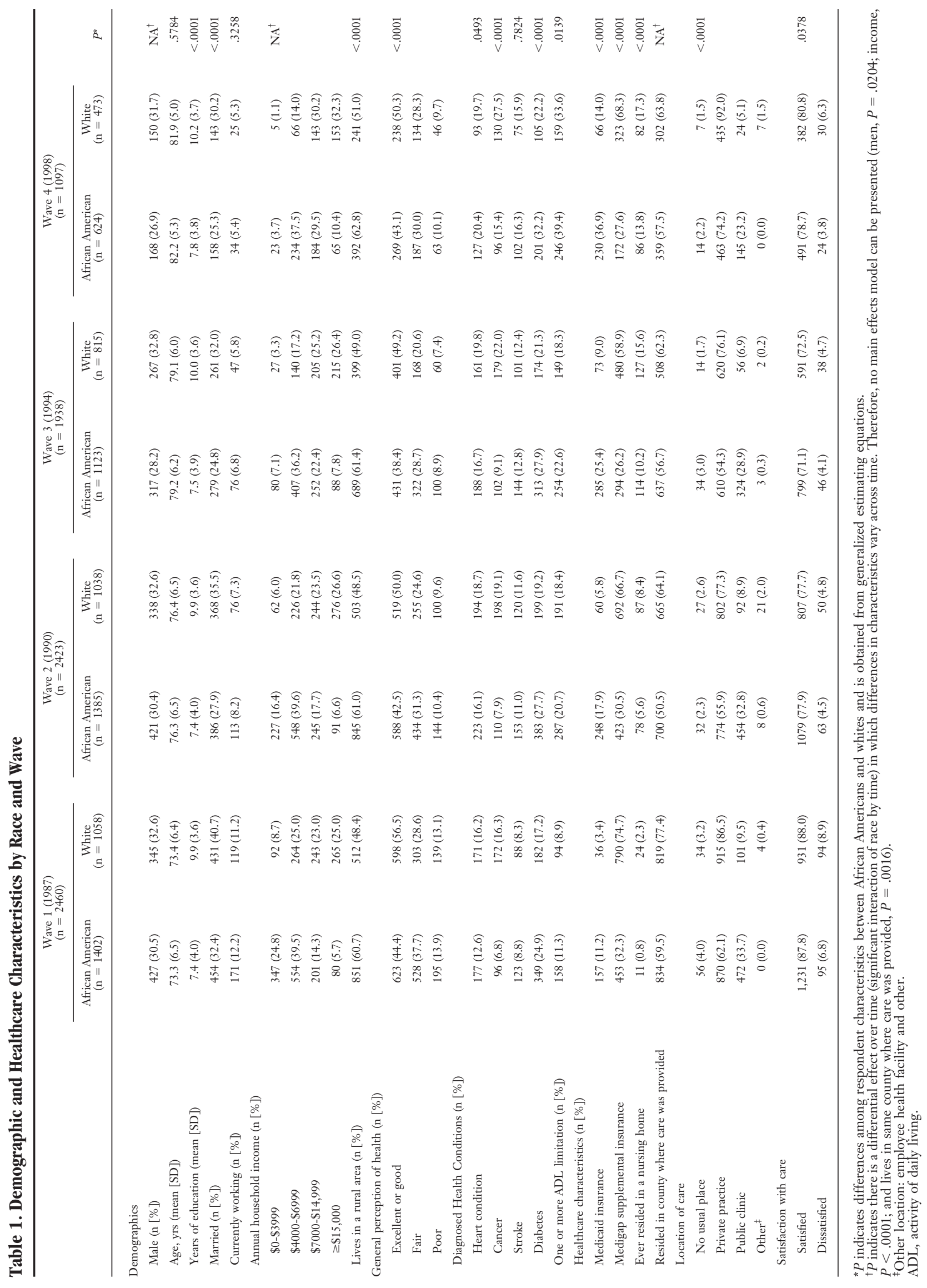


Table 2. Distribution of Consistency of Care by Race and Wave

\begin{tabular}{|c|c|c|c|c|c|c|c|c|c|}
\hline \multirow[b]{2}{*}{$\begin{array}{l}\text { Consistency of } \\
\text { Care (n }[\%])\end{array}$} & \multicolumn{2}{|c|}{$\begin{array}{c}\text { Wave } 1(1987) \\
\quad(\mathrm{n}=2460)\end{array}$} & \multicolumn{2}{|c|}{$\begin{array}{l}\text { Wave } 2(1990) \\
\quad(\mathrm{n}=2423)\end{array}$} & \multicolumn{2}{|c|}{$\begin{array}{c}\text { Wave } 3(1994) \\
(\mathrm{n}=1938)\end{array}$} & \multicolumn{2}{|c|}{$\begin{array}{c}\text { Wave } 4(1998) \\
\quad(\mathrm{n}=1097)\end{array}$} & \multirow[b]{2}{*}{$P^{*}$} \\
\hline & $\begin{array}{c}\text { African } \\
\text { American } \\
(\mathrm{n}=1402)\end{array}$ & $\begin{array}{c}\text { White } \\
(\mathrm{n}=1058)\end{array}$ & $\begin{array}{c}\text { African } \\
\text { American } \\
(\mathrm{n}=1385)\end{array}$ & $\begin{array}{c}\text { White } \\
(\mathrm{n}=1038)\end{array}$ & $\begin{array}{c}\text { African } \\
\text { American } \\
(\mathrm{n}=1123)\end{array}$ & $\begin{array}{c}\text { White } \\
(\mathrm{n}=815)\end{array}$ & $\begin{array}{c}\text { African } \\
\text { American } \\
(\mathrm{n}=624)\end{array}$ & $\begin{array}{c}\text { White } \\
(n=473)\end{array}$ & \\
\hline Consistent care & $1120(79.9)$ & $956(90.4)$ & $838(60.5)$ & $773(74.5)$ & $603(53.7)$ & $551(67.6)$ & $381(61.1)$ & $362(76.5)$ & $<.0001$ \\
\hline Inconsistent care $^{\dagger}$ & - & - & $381(27.5)$ & $214(20.6)$ & $454(40.4)$ & $250(30.7)$ & $224(35.9)$ & $106(22.4)$ & $<.0001$ \\
\hline No standard care & $282(20.1)$ & $102(9.6)$ & $166(12.0)$ & $51(4.9)$ & $66(5.9)$ & $14(1.7)$ & $19(3.0)$ & $5(1.1)$ & $<.0001$ \\
\hline
\end{tabular}

*Two-sided test of racial difference in consistency of care characteristics over time using generalized estimating equations.

${ }^{\dagger}$ Inconsistent care was not assessed at wave 1 because the definition requires data from 2 or more time periods.

waves and for both racial groups, the mean systolic $\mathrm{BP}$ was $>140 \mathrm{~mm} \mathrm{Hg}$ whereas the mean diastolic BP was $<90 \mathrm{~mm} \mathrm{Hg}$ (Table 3). No racial differences were noted for systolic BP, but African Americans had a higher diastolic BP $(P<.001)$ compared with whites. There was an increase over time from wave 1 to wave 4 in the proportion of African Americans and whites with controlled BP. Over all 4 waves, a greater proportion of whites had stage 1 hypertension although there was no significant difference in the proportions of those with stage 2 hypertension.

The distribution of consistent care and BP control by wave is presented in Table 4 and the odds ratios and $95 \% \mathrm{CI}$ for the association between consistent care and BP control are presented in Table 5. In crude analyses, participants with consistent care or inconsistent care had greater odds of BP control (odds ratio [OR] 1.51; 95\% CI, 1.241.84 and OR, 2.08; 95\% CI, 1.67-2.59, respectively) compared with those with no standard care. Additional adjustment for sociodemographic factors slightly attenuated the association for consistent (OR, 1.26; 95\% CI, 0.99-1.59) and inconsistent care (OR, 1.35; 95\% CI, 1.03-1.77). Inclusion of insurance, satisfaction with medical care, and health history variables further attenuated these associations. Effect modification by race was not significant, nor were there differences in BP control after adjustment for sociodemographic and health care variables (OR, 1.06; 95\% CI, 0.901.24).

\section{Discussion}

In this population-based, observational study of hypertensive, elderly African Americans and whites, participants with consistent care or inconsistent care had greater odds of BP control compared with participants with no standard care during a 12-year period; however, these associations were attenuated after adjustment for sociodemographic and health care characteristics.

A previous study reported that consistent care was associated with recognition of hypertension and receipt of antihypertensive therapy among the elderly, ${ }^{7}$ whereas another study reported no association between consistent care and recognition of hypertension after adjustment for potential confounders. ${ }^{11}$ These findings for hypertension awareness could have implications for BP treatment and control, particularly among the elderly given that hypertension prevalence increases with age. In this prospective study of the elderly, an association between consistency of care and BP control was found, but this association was explained by health care characteristics and comorbidities. This study's results are similar to findings from a cross-sectional study that reported that using the same health care facility or the same health care provider was associated with BP control among the general population, although that study's association persisted even after adjustment for covariates. ${ }^{5}$

Inconsistent care and no standard care were more common among African Americans than white participants in this study. No racial differences in BP control were found, but the differences in receipt of physician care are notable given that African Americans had poorer demographic and health care characteristics. These adverse characteristics observed over time among African Americans as compared with whites may affect health care outcomes, including BP control, which requires long-term management. In our study, African Americans were significantly less likely than whites to have private supplemental health insurance, which helps to defray out-of-pocket costs; 


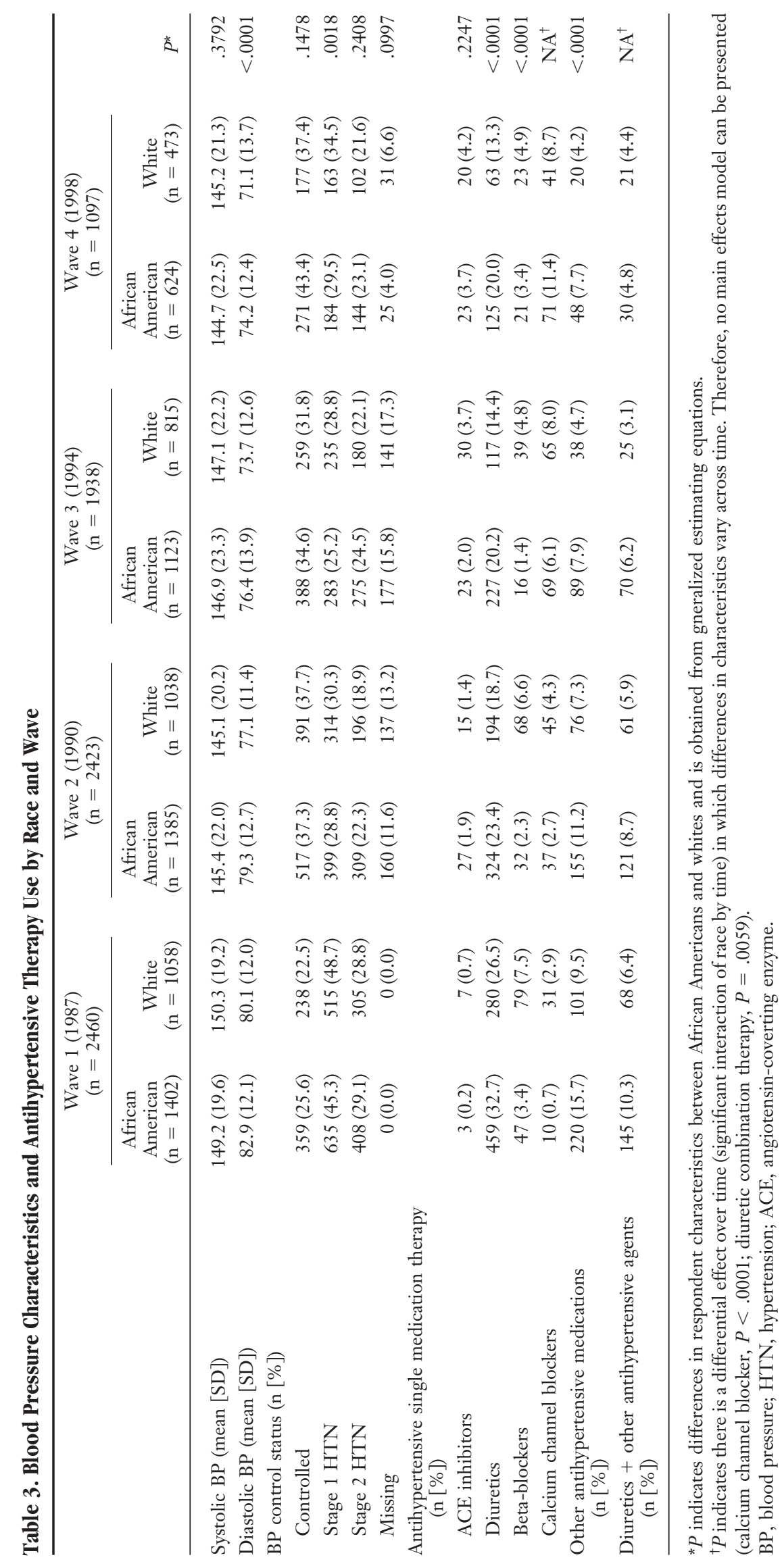




\begin{tabular}{|c|c|c|}
\hline Model* & Variable & $\begin{array}{l}\text { Odds Ratio } \\
\text { (95\% CI) }\end{array}$ \\
\hline \multirow[t]{3}{*}{1} & No standard care & 1.00 \\
\hline & Inconsistent care & $2.08(1.67,2.59)^{\dagger}$ \\
\hline & Consistent care & $1.51(1.24,1.84)^{\dagger}$ \\
\hline \multirow[t]{4}{*}{2} & No standard care & 1.00 \\
\hline & Inconsistent care & $2.09(1.68,2.60)^{\dagger}$ \\
\hline & Consistent care & $1.54(1.26,1.88)^{\dagger}$ \\
\hline & African American race & $1.10(0.98,1.23)$ \\
\hline \multirow[t]{4}{*}{3} & No standard care & 1.00 \\
\hline & Inconsistent care & $1.41(1.12,1.78)^{\ddagger}$ \\
\hline & Consistent care & $1.34(1.09,1.64)^{\ddagger}$ \\
\hline & African American race & $1.10(0.98,1.24)^{\S}$ \\
\hline \multirow[t]{4}{*}{4} & No standard care & 1.00 \\
\hline & Inconsistent care & $1.35(1.03,1.77)^{\|}$ \\
\hline & Consistent care & $1.26(0.99,1.59)^{\S}$ \\
\hline & African American race & $1.04(0.90,1.19)$ \\
\hline \multirow[t]{4}{*}{5} & No standard care & 1.00 \\
\hline & Inconsistent care & $1.14(0.85,1.52)$ \\
\hline & Consistent care & $1.00(0.78,1.28)$ \\
\hline & African American race & $1.06(0.90,1.24)$ \\
\hline
\end{tabular}

*Model 1, unadjusted; model 2, adjusted for race; model 3, adjusted for race, age at baseline, gender, and study year; model 4 , adjusted for race, age at baseline, gender, and study year, marital status, work status, education, and income; model 5, adjusted for race, age at baseline, gender, and study year, marital status, work status, education, income, residence in a rural area, nursing home residence, Medicaid insurance, Medigap supplemental insurance, satisfaction with medical care, physical limitation, use of antihypertensive medication, and history of stroke, diabetes, heart disease, and cancer.

${ }^{\dagger} P<.0001 ;{ }^{\ddagger} P<.01 ;{ }^{\|} P<.05 ;{ }^{\circledR} P<.10$.

lack of financial resources may be a barrier to hypertension management. ${ }^{12}$ Furthermore, African Americans' lack of satisfaction with previous care received may contribute to a lack of trust in the health care system and also deter health care-seeking behaviors. ${ }^{13-15}$

This study has several limitations. The PHSE study collected information about study participants during 4 visits during 12 years. Consistent care and BP control were only assessed during these visits, so it is possible that participants had breaks in care or fluctuated between controlled and uncontrolled BP, which could not be evaluated in this study. Also, the definition of consistent care was limited here because participants were asked where they usually seek care and to name their physician. The definition of consistency of care 


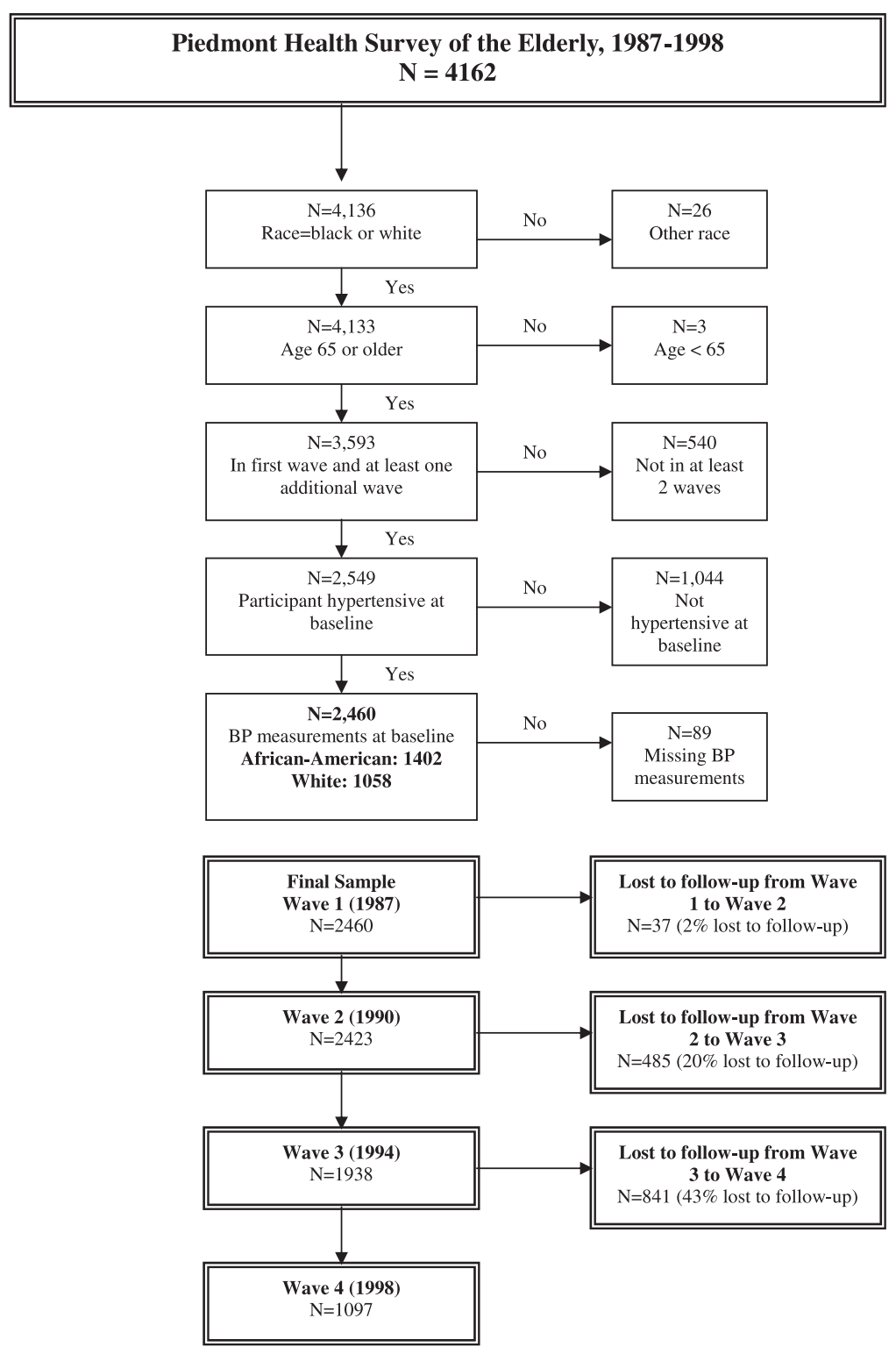

Figure 1. BP, blood pressure.

used is just one measure of the physician-patient care relationship. Other measures have been reported in the literature, such as continuity of care, usual source of care, and usual provider of care, and may be more specific than the definition used here because of the use of various indices, such as the usual provider of care index, which measures the number of visits to a usual provider divided by the total number of primary care visits. ${ }^{16}$ Consistent care is difficult to define because it is typically used to assess the various aspects of the interpersonal relationship between the physician and the patient ${ }^{17,18}$ in addition to encompassing other attributes such as the extent of the visit, knowledge, and environment. ${ }^{19}$ The definition of consis- tency of care in this study did not assess these different aspects of the physician-patient care relationship, so some attributes of the relationship may not be captured in the definition used. Also, this study did not have information about medication adherence by participants, which is associated with $\mathrm{BP}$ control and may be affected by consistency of care. Another potential limitation to this study is the high attrition rate cause by the advanced age of the participants. Attrition and missing data could bias results if they are not random, but there was no difference observed in a supplemental analysis. Also, the participants were mostly from rural areas of North Carolina, so these results may not be generalizable to other populations. 


\section{Conclusions}

Consistent and inconsistent care were associated with greater odds of BP control compared with no standard care in this prospective study of the elderly. AfricanAmerican participants disproportionately received inconsistent or no standard care during the 12-year study period, although no racial differences were noted in $\mathrm{BP}$ control over time. Future studies should continue to explore the complexity of medical care relationships to identify factors that may affect $\mathrm{BP}$ control among the elderly.

\section{References}

1. Cooper R, Rotimi C. Hypertension in blacks. Am J Hypertens 1997;10(7 Pt 1):804-12.

2. Hajjar I, Kotchen TA. Trends in prevalence, awareness, treatment, and control of hypertension in the United States, 1988-2000. JAMA 2003;290:199206.

3. McGruder HF, Malarcher AM, Antoine TL, Greenlunch KJ, Croft JB. Racial and ethnic disparities in cardiovascular risk factors among stroke survivors: United States 1999 to 2001. Stroke 2004;35:1557-61.

4. Chobanian AV, Bakris GL, Black HR, et al. Seventh report of the Joint National Committee on Prevention, Detection, Evaluation, and Treatment of High Blood Pressure. Hypertension 2003;42:1206-52.

5. He J, Muntner P, Chen J, Roccella EJ, Streiffer RH, Whelton PK. Factors associated with hypertension control in the general population of the United States. Arch Intern Med 2002;162:1051-8.

6. Saultz JW. Continuity of care. In: Textbook of Family Medicine. J.W. Saultz (ed). New York: McGrawHill; 2000:52-77.

7. Konrad TR, Howard DL, Edwards LJ, Ivanova A, Carey TS. Physician-patient racial concordance, continuity of care, and patterns of care for hypertension. Am J Public Health 2005;95:2186-90.

8. Psaty BM, Manolio TA, Smith NL, et al. Time trends in high blood pressure control and the use of antihypertensive medications in older adults: the Cardiovascular Health Study. Arch Intern Med 2002;162:2325-32.

9. Cornoni-Huntley J, Ostfeld AM, Taylor JO, et al. Established populations for epidemiologic studies of the elderly: study design and methodology. Aging (Milano) 1993;5:27-37.

10. Diggle PJ, Heagarty P, Liang KY, et al., Analysis of longitudinal data, 2nd ed. New York: Oxford Press; 2002.

11. Koopman RJ, Mainous AG 3rd, Baker R, Gill JM, Gilbert GE. Continuity of care and recognition of diabetes, hypertension, and hypercholesterolemia. Arch Intern Med 2003;163:1357-61.

12. Coleman MT, Lott JA, Sharma S. Use of continuous quality improvement to identify barriers in the management of hypertension. Am J Med Qual 2000;15: $72-7$.

13. Armstrong K, McMurphy S, Dean LT, et al. Differences in the patterns of health care system distrust between blacks and whites. J Gen Intern Med 2008; 23:827-33.

14. Blanchard J, Lurie N. R-E-S-P-E-C-T: patient reports of disrespect in the health care setting and its impact on care. J Fam Pract 2004;53:721-30.

15. Blanchard J, Nayar S, Lurie N. Patient-provider and patient-staff racial concordance and perceptions of mistreatment in the health care setting. J Gen Intern Med 2007;22:1184-9.

16. Breslau N, Reeb KG. Continuity of care in a university-based practice. J Med Educ 1975;50:965-9.

17. Saultz JW. Defining and measuring interpersonal continuity of care. Ann Fam Med 2003;1:134-43.

18. Saultz JW, Lochner J. Interpersonal continuity of care and care outcomes: a critical review. Ann Fam Med 2005;3:159-66.

19. Rogers J, Curtis P. The concept and measurement of continuity in primary care. Am J Public Health 1980; $70: 122-7$. 